\title{
Model reduction of time-delay systems using position balancing and delay Lyapunov equations
}

\author{
Elias Jarlebring \\ Tobias Damm \\ Wim Michiels
}

Report TW602, October 2011

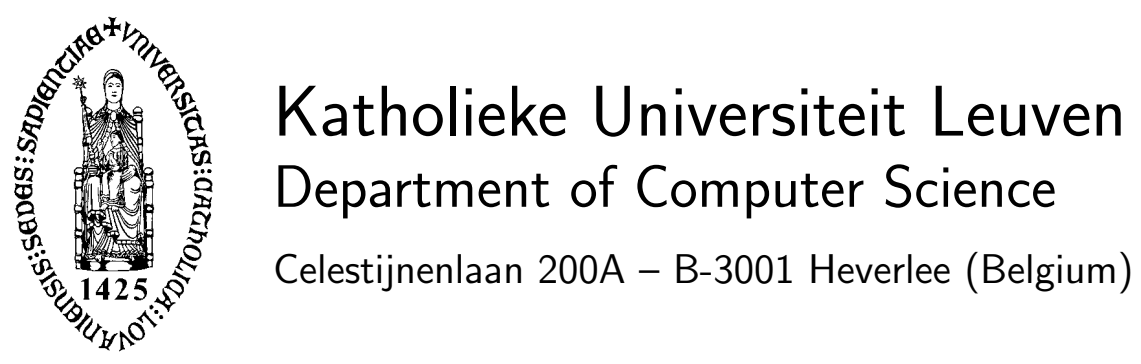




\title{
Model reduction of time-delay systems using position balancing and delay Lyapunov equations
}

\author{
Elias Jarlebring \\ Tobias Damm \\ Wim Michiels
}

Report TW602, October 2011

Department of Computer Science, K.U.Leuven

\begin{abstract}
Balanced truncation is a standard and very natural approach to approximate dynamical systems. We present a version of balanced truncation for model order reduction of linear time-delay systems. The procedure is based on a coordinate transformation of the position and preserves the delay structure of the system. We therefore call it (structure preserving) position balancing. To every position we associate quantities representing energies for the controllability and observability of the position. We show that these energies can be expressed explicitly in terms of the solutions to corresponding delay Lyapunov equations. Apart from characterizing the energies, we show that one block of the (operator) controllability and observability Gramians in the operator formulation of the time-delay system can also be characterized with the delay Lyapunov equation. The delay Lyapunov equation undergoes a contragredient transformation when we apply the position coordinate transformation and we propose to truncate it in a classical fashion, such that positions which are only weakly connected to the input and the output in the sense of the energy concepts are removed.
\end{abstract}

Keywords : Time-delay systems, Lyapunov equations, Model reduction. 
manuscript No.

(will be inserted by the editor)

\title{
Model reduction of time-delay systems using position balancing and delay Lyapunov equations
}

\author{
Elias Jarlebring, Tobias Damm, Wim Michiels
}

the date of receipt and acceptance should be inserted later

\begin{abstract}
Balanced truncation is a standard and very natural approach to approximate dynamical systems. We present a version of balanced truncation for model order reduction of linear time-delay systems. The procedure is based on a coordinate transformation of the position and preserves the delay structure of the system. We therefore call it (structure preserving) position balancing. To every position we associate quantities representing energies for the controllability and $o b$ servability of the position. We show that these energies can be expressed explicitly in terms of the solutions to corresponding delay Lyapunov equations. Apart from characterizing the energies, we show that one block of the (operator) controllability and observability Gramians in the operator formulation of the time-delay system can also be characterized with the delay Lyapunov equation. The delay Lyapunov equation undergoes a contragredient transformation when we apply the position coordinate transformation and we propose to truncate it in a classical fashion, such that positions which are only weakly connected to the input and the output in the sense of the energy concepts are removed.
\end{abstract}

\section{Introduction}

Models consisting of dynamical systems with a delay arise naturally when studying phenomena involving events occuring in a non-instantaneous manner. Here, we

E. Jarlebring

K.U. Leuven, Department of Computer Science, 3001 Heverlee, Belgium,

E-Mail: elias.jarlebring@cs.kuleuven.be

T. Damm

Universität Bayreuth, Mathematisches Institut, Universitässtraße 30, D-95440 Bayreuth, Germany, E-Mail: tobias.damm@uni-bayreuth.de

W. Michiels

K.U. Leuven, Department of Computer Science, 3001 Heverlee, Belgium,

E-Mail: wim.michiels@cs.kuleuven.be 
consider a linear time-invariant time-delay system with a single delay,

$$
\begin{aligned}
& \dot{x}(t)=A_{0} x(t)+A_{1} x(t-\tau)+B_{0} u(t) \\
& y(t)=C_{0} x(t),
\end{aligned}
$$

where $A_{0}, A_{1} \in \mathbb{R}^{n \times n}, B_{0} \in \mathbb{R}^{n \times m}, C_{0}^{\mathrm{T}} \in \mathbb{R}^{n \times p}$; see the standard references on time-delay systems [9], [25],[23].

If, for example, the time-delay system stems from a discretization of a partial differential equation, then the order $n$ can be so large that it is computationally difficult to solve or analyze the problem. In these situations, it is often advantageous to approximate (1) by a system of smaller size. Such an approach of model order reduction is well-established for dynamical systems without delay, cf. [1], [2], [5].

The fact that (1) is a time-delay system has several consequences in terms of qualitative physical properties. Some of these properties will be lost in the approximation unless the reduced system also has a delay structure. In this paper we construct an algorithm to compute a model where the structure is preserved, i.e., the reduced system will also be a linear time-invariant time-delay system of the form

$$
\begin{aligned}
& \dot{\hat{x}}(t)=\hat{A_{0}} \hat{x}(t)+\hat{A_{1}} \hat{x}(t-\tau)+\hat{B}_{0} u(t) \\
& y(t)=\hat{C}_{0} \hat{x}(t)
\end{aligned}
$$

with $\hat{A}_{0}, \hat{A}_{1} \in \mathbb{R}^{r \times r}, \hat{B}_{0} \in \mathbb{R}^{r \times m}, \hat{C}_{0}^{\mathrm{T}} \in \mathbb{R}^{r \times p}$ and $r \ll n$.

A number of approaches for model reduction of time-delay systems are available in the literature. For instance, there are methods with interpretation as rational approximation [19], [20], methods based on interpolation [4], Krylov methods in an infinite dimensional setting [10], moment matching based methods [24], and methods based on the dominance of poles [32]. Some open problems related to model reduction of time-delay systems are formulated in [29]. To our knowledge, no structure preserving variants of balanced truncation are available for time-delay systems.

Our approach is based on associating, with each position, controllability and observability functionals and we characterize them with the help of Gramians. While in the case of delay-free systems these Gramians are obtained from algebraic Lyapunov equations, here we will have to consider delay Lyapunov equations. The solutions of the delay Lyapunov equations related to controllability and observability energies will be denoted by $U_{c}:[-\tau, \tau] \rightarrow \mathbb{R}^{n \times n}$ and $U_{o}:[-\tau, \tau] \rightarrow \mathbb{R}^{n \times n}$, respectively. The matrices $U_{c}(0)$ and $U_{o}(0)$ are symmetric nonnegative definite and the corresponding quadratic forms characterize the controllability and observability energies associated to given a position.

Moreover, we show that $U_{c}(0)$ and $U_{o}(0)$ can be interpreted as submatrices of the infinite-dimensional Gramians associated to the full state of the time-delay system. This is in analogy to the case of second-order systems (e.g. [7]), which we discuss as a motivating and illustrating class of systems.

By considering a linear transformation of the position, we can achieve balancing of $U_{c}(0)$ and $U_{o}(0)$. This suggests to carry out the model reduction by truncating those positions which are both hard to reach and hard to observe, in the sense of the derived energy concepts. 


\section{Preliminaries}

2.1 The fundamental solution and delay Lyapunov equations

The fundamental solution corresponding to (1) is given by the matrix delay-differential equation

$$
\begin{aligned}
\dot{K}(t) & =A_{0} K(t)+A_{1} K(t-\tau) \\
K(0) & =I, \quad K(\theta)=0 \text { when } \theta<0 .
\end{aligned}
$$

Suppose the system (1) is exponentially stable. Then, the fundamental solution decays exponentially, and we can define a finite parameter-dependent matrix,

$$
U_{o}(\theta):=\int_{0}^{\infty} K(t)^{\mathrm{T}} C_{0}^{\mathrm{T}} C_{0} K(t+\theta) d t
$$

which is called a delay Lyapunov matrix. It is related to the observability problem for the delay system; more precisely to an energy quantity representing observability, as we shall explain in Section 3.1. Further properties of $U_{o}$ have been studied, characterized and used in a number of settings. The existance of a solution and how it can be used to study stability with Lyapunov-Krasovskii functionionals is given in different generality settings in [15],[13],[18],[17],[34],[14]. It has been used to derive a bound on the solution of (1) in [16]. Some computational aspects are considered in [26], [11], [27] and it has been used to compute the $\mathcal{H}_{2}$ norm of (1) in $[12]$.

In particular (cf. [17]) the function $U_{o}:[-\theta, \theta] \rightarrow \mathbb{R}^{n \times n}$ is the unique solution of the boundary value problem

$$
\begin{aligned}
U_{o}^{\prime}(t) & =U_{o}(t) A_{0}+U_{o}(t-\tau) A_{1}, t \geq 0 \\
U_{o}(-t) & =U_{o}(t)^{\mathrm{T}} \\
-C_{0}^{\mathrm{T}} C_{0} & =U_{o}(0) A_{0}+A_{0}^{\mathrm{T}} U_{o}(0)+U_{o}(-\tau) A_{1}+A_{1}^{\mathrm{T}} U_{o}(\tau) .
\end{aligned}
$$

This characterization of $U_{o}$ is useful both for theoretical and computational reasons and gives rise to an explicit solution in terms of a matrix exponential [30], [12].

The dual delay Lyapunov matrix related to controllability is given by

$$
U_{c}(\theta):=\int_{0}^{\infty} K(t) B_{0} B_{0}^{\mathrm{T}} K(t+\theta)^{\mathrm{T}} d t .
$$

Following the steps to derive (4) in [17] it is straightforward to show that $U_{c}$ also satisfies a similar matrix boundary value problem,

$$
\begin{aligned}
U_{c}^{\prime}(t) & =U_{c}(t) A_{0}^{\mathrm{T}}+U_{c}(t-\tau) A_{1}^{\mathrm{T}}, t \geq 0 \\
U_{c}(-t) & =U_{c}(t)^{\mathrm{T}} \\
-B_{0} B_{0}^{\mathrm{T}} & =U_{c}(0) A_{0}^{\mathrm{T}}+A_{0} U_{c}(0)+U_{c}(-\tau) A_{1}^{\mathrm{T}}+A_{1} U_{c}(\tau) .
\end{aligned}
$$

Note that (4) and (6) correspond to transposition of the system matrices and switching the roles of $B_{0}$ and $C_{0}^{\mathrm{T}}$. This is consistent with the notion of dual timedelay system used, e.g. in [21]. It is also a consistent generalization of the controllability and observability Gramians of a dynamical system without delay.

We also need the fact that $U_{c}(0)$ and $U_{o}(0)$ are symmetric positive semidefinite. This follows from the fact that the integrand in the definition of $U_{c}(0)$ and $U_{o}(0)$ are symmetric positive semidefinite. 


\subsection{Position balancing}

The concept of position balancing is well-established for second order systems and it is used in many variants of balanced truncation [6], [7], [22],[31], [35]. To prepare its modification for time-delay systems, we briefly review the idea of position balancing for second order systems, following the reasoning in [7],[22],[31].

Consider an exponentially stable second order system

$$
\begin{aligned}
& M \ddot{x}(t)+G \dot{x}+K x=B u(t), \quad x(t) \in \mathbb{R}^{n}, \\
& y(t)=C_{1} x(t)+C_{2} \dot{x}(t),
\end{aligned}
$$

where $M$ is nonsingular. In the context of mechanical systems, $x$ is usually referred to as the position and $\dot{x}=: v$ as the velocity. For given initial values $x(0)=x_{0}$ and $v(0)=v_{0}$ and a given input function $u$ we denote the corresponding solutions by $x\left(t, x_{0}, v_{0}, u\right)$ and $v\left(t, x_{0}, v_{0}, u\right)$, and the output by $y\left(t, x_{0}, v_{0}, u\right)$.

The second order system can be written in first-order form as

$$
\begin{aligned}
{\left[\begin{array}{c}
\dot{x}(t) \\
\dot{v}(t)
\end{array}\right] } & =\left[\begin{array}{cc}
0 & I \\
-M^{-1} K-M^{-1} G
\end{array}\right]\left[\begin{array}{l}
x(t) \\
v(t)
\end{array}\right]+\left[\begin{array}{c}
0 \\
B
\end{array}\right] u(t), \\
y(t) & =C_{1} x(t)+C_{2} v(t) .
\end{aligned}
$$

Let $P=P^{\mathrm{T}} \in \mathbb{R}^{2 n \times 2 n}$ and $Q=Q^{\mathrm{T}} \in \mathbb{R}^{2 n \times 2 n}$ denote the controllability and observability Gramians of (8). By definition $P$ and $Q$ are nonnegative definite, and for simplicity, we assume that $P$ is nonsingular. Then the total energy flowing out of the uncontrolled system is given by

$$
\int_{0}^{\infty}\left\|y\left(t, x_{0}, v_{0}, 0\right)\right\|^{2} d t=\left(\begin{array}{c}
x_{0} \\
v_{0}
\end{array}\right)^{\mathrm{T}} Q\left(\begin{array}{l}
x_{0} \\
v_{0}
\end{array}\right)
$$

and the minimal control energy needed to reach a state $\left(x_{1}, v_{1}\right)$ asymptotically from

$$
x(0)=0, v(0)=0,
$$

is given by

$$
\inf _{\substack{T>0, u \in L_{2}([0, T]) \\
x(T, 0,0, u)=x_{1} \\
v(T, 0,0, u)=v_{1}}} \int_{0}^{T}\|u(t)\|^{2} d t \rightarrow\left(\begin{array}{l}
x_{1} \\
v_{1}
\end{array}\right)^{\mathrm{T}} P^{-1}\left(\begin{array}{l}
x_{1} \\
v_{1}
\end{array}\right) .
$$

We call the system balanced if $P=Q=\Sigma$ is diagonal. In this case every state is equally difficult to reach as it is to observe. Balancing can be achived with a coordinate transformation

$$
\left(\begin{array}{l}
\tilde{x} \\
\tilde{y}
\end{array}\right)=R\left(\begin{array}{l}
x \\
y
\end{array}\right)
$$

which results in a contragredient transformation of the Gramians $P$ and $Q$ (see e.g. [36]). Note that the transformation of the state (11) does in general result in a dynamical system of size $2 n \times 2 n$, but not of the form (8), i.e., the transformation destroys the second order structure. To avoid this we consider only transformations of the position

$$
\tilde{x}=T x
$$


This restriction has the consequence that in general we can not balance the full matrices $Q$ and $P$. If we partition

$$
\left[\begin{array}{ll}
Q_{11} & Q_{12} \\
Q_{21} & Q_{22}
\end{array}\right]:=Q, \quad\left[\begin{array}{ll}
P_{11} & P_{12} \\
P_{21} & P_{22}
\end{array}\right]:=P,
$$

it is easy to show that the transformation (12) induces a contragredient transformation of the blocks $P_{11}$ and $Q_{11}$ and the transformation (12) does provide enough freedom to balance those blocks in in the Gramians.

Definition 1 The second order system (7) is called position-balanced if

$$
P_{11}=Q_{11}=\Sigma,
$$

where $\Sigma$ is diagonal.

The restricted Gramians $Q_{11}$ and $P_{11}$ describe the observability and reachability energies of the positions (cf. [22]). Namely, it follows from (9) that

$$
\int_{0}^{\infty}\left\|y\left(t, x_{0}, 0,0\right)\right\|^{2} d t=x_{0}^{\mathrm{T}} Q_{11} x_{0} .
$$

By an inversion formula for block matrices (e.g. [28, Thm. 2.7]) we have

$$
P^{-1}=\left[\begin{array}{cc}
P_{11}^{-1} & 0 \\
0 & 0
\end{array}\right]+\left[\begin{array}{c}
P_{11}^{-1} P_{12} \\
-I
\end{array}\right] S^{-1}\left[\begin{array}{c}
P_{11}^{-1} P_{12} \\
-I
\end{array}\right]^{*}
$$

with $S=P_{22}-P_{12}^{*} P_{11}^{-1} P_{12}>0$. Applying this to (10) we obtain

$$
\inf _{\substack{T>0, u \in L_{2}([0, T]) \\ x(T, 0,0, u)=x_{1}}} \int_{0}^{T}\|u(t)\|^{2} d t=x_{1}^{\mathrm{T}} P_{11}^{-1} x_{1},
$$

where the optimal limiting velocity is $v(T, 0,0, u)=v_{1}=P_{12}^{*} P_{11}^{-1} x_{1}$.

Remark 1 The position transformation (12) preserves the second order structure but only involves a restricted class of transformations of the state. This changes the energy concepts in the sense that we associate the energies with a given position instead of a state. The observability energy is the energy associated with starting with zero velocity and the controllability energy is the energy associated with the optimization problem where the final velocity is also optimized. In [22] the optimization problem (16) is referred to as the free velocity optimization problem.

\section{Position balancing of time-delay systems}

In order to construct a balancing procedure which preserves the structure of the time-delay system (1), we will now, inspired by the second order case, consider a transformation of the position,

$$
\tilde{x}=T x \text {. }
$$

We derive an analogous concept of position balancing, where now the delay Lyapunov matrices at zero, $U_{c}(0)$ and $U_{o}(0)$, play the same role as the matrices $P_{11}$ and $Q_{11}$ in the previous section.

First, note that (17) induces a contragredient transformation of $U_{c}(0)$ and $U_{o}(0)$. The following result follows directly from the equations (6) and (4). 
Lemma 1 (Contragredient transformation) Consider an exponentially stable timedelay system defined by (1) and the associated delay Lyapunov matrices $U_{c}$ and $U_{o}$. The time-delay system defined by the coordinate transformation (17) is given by $\tilde{A}_{0}=$ $T A_{0} T^{-1}, \tilde{A}_{1}=T A_{1} T^{-1}, \tilde{B}_{0}=T B_{0}$ and $\tilde{C}_{0}=C_{0} T^{-1}$. Moreover, the associated delay Lyapunov matrices satisfy

$$
\tilde{U}_{o}(0)=T^{-\mathrm{T}} U_{o}(0) T^{-1}, \quad \tilde{U}_{c}(0)=T U_{c}(0) T^{\mathrm{T}} .
$$

3.1 Energy functionals and delay Lyapunov equations

Consider a time-delay system (1) with $x(0)=x_{0}$ and $x(t)=\varphi_{0}(t)$ for $-\tau \leq$ $t<0$. For a given input $u$, we will denote the position and output of such a system by $x\left(t, x_{0}, \varphi_{0}, u\right):=x(t)$ and $y\left(t, x_{0}, \varphi_{0}, u\right):=y(t)$. In analogy to (14), the (observability) energy associated with the position $x_{0}$ can be defined as

$$
E_{o}\left(x_{0}, T\right):=\int_{0}^{T}\left\|y\left(t, x_{0}, 0,0\right)\right\|^{2} d t .
$$

Since $x(t)=K(t) x_{0}$ and $y(t)=C_{0} x(t)=C_{0} K(t) x_{0}$, we have $E_{o}\left(x_{0}, T\right) \rightarrow x_{0}^{\mathrm{T}} U_{o}(0) x_{0}$ as $T \rightarrow \infty$. We have hence expressed the energy (18) for $T \rightarrow \infty$ in terms of $U_{o}(0)$.

In analogy to (16), we consider the minimal energy needed to steer the system (at rest at $t=0$ ) to a given position $x_{1}$ in time $T$ as

$$
E_{c}\left(x_{1}, T\right):=\min _{\substack{u \in L_{2}([0, T]), x(T, 0,0, u)=x_{1}}} \int_{0}^{T}\|u(t)\|^{2} d t .
$$

In order to characterize the minimum and its limit for $T \rightarrow \infty$ we need an explicit expression for the optimal control $u$. The optimal control is given in the following lemma, where $(\cdot)^{\dagger}$ denotes the Moore-Penrose inverse.

Lemma 2 (Optimal control) Consider an exponentially stable time-delay system (1), with a fundamental solution $K$. Moreover, let

$$
P(\theta):=\int_{0}^{\theta} K(\theta-s) B_{0} B_{0}^{\mathrm{T}} K(\theta-s)^{\mathrm{T}} d s .
$$

If $x_{1} \in \operatorname{Im} P(T)$, then the control $u_{x_{1}}:[0, T] \rightarrow \mathbb{R}^{m}$ defined by

$$
u_{x_{1}}(t)=B_{0}^{\mathrm{T}} K(T-t)^{\mathrm{T}} P(T)^{\dagger} x_{1}
$$

is the unique minimizer in (19) and

$$
E_{c}\left(x_{1}, T\right)=\left\|u_{x_{1}}\right\|_{L^{2}}=x_{1}^{\mathrm{T}} P(T)^{\dagger} x_{1} .
$$

Proof Inserting $x_{0}=0$ and $u_{x_{1}}$ in the variation-of-constants formula, we have

$$
x\left(T, 0, u_{x_{1}}\right)=\int_{0}^{T} K(T-s) B_{0} B_{0}^{\mathrm{T}} K(T-s)^{\mathrm{T}} P(T)^{\dagger} x_{1} d s=P(T) P(T)^{\dagger} x_{1}=x_{1},
$$


and

$$
\begin{aligned}
\int_{0}^{T}\left\|u_{x_{1}}(t)\right\|^{2} d t & =\int_{0}^{T} x_{1}^{\mathrm{T}} P(T)^{\dagger} K(T-t) B_{0} B_{0}^{\mathrm{T}} K(T-t)^{\mathrm{T}} P(T)^{\dagger} x_{1} d t \\
& =x_{1}^{\mathrm{T}} P(T)^{\dagger} P(T) P(T)^{\dagger} x_{1}=x_{1}^{\mathrm{T}} P(T)^{\dagger} x_{1} .
\end{aligned}
$$

It remains to prove that $u_{x_{1}}$ is optimal. So let $\tilde{u}$ be another input satisfying $x(T, 0,0, \tilde{u})=x_{1}$. Taking the difference of both variation-of-constants representations, we find that

$$
\int_{0}^{T} K(T-s) B_{0}\left(\tilde{u}(s)-u_{x_{1}}(s)\right) d s=0
$$

and thus the orthogonality relation $\int_{0}^{T} u_{x_{1}}(s)^{\mathrm{T}}\left(\tilde{u}(s)-u_{x_{1}}(s)\right) d s=0$. By the Pythagorean theorem we have

$$
\|\tilde{u}\|_{L^{2}}^{2}=\left\|u_{x_{1}}+\left(\tilde{u}-u_{x_{1}}\right)\right\|_{L^{2}}^{2}=\left\|u_{x_{1}}\right\|_{L^{2}}^{2}+\left\|\left(\tilde{u}-u_{x_{1}}\right)\right\|_{L^{2}}^{2},
$$

showing that $\|\tilde{u}\|_{L^{2}} \geq\left\|u_{x_{1}}\right\|_{L^{2}}$ with equality only for $\tilde{u}=u_{x_{1}}$

Now note that by variable substitution and equation (5) we have

$$
P(T)=\int_{0}^{T} K(t) B_{0} B_{0}^{\mathrm{T}} K(t)^{\mathrm{T}} d t \rightarrow U_{c}(0) \text { as } T \rightarrow \infty .
$$

Thus, we have also characterized (19) in terms of the solution to the delay Lyapunov equation. We summarize these results in the following theorem.

Theorem 1 Consider an exponentially stable time-delay system (1) and let $U_{c}$ and $U_{o}$ be the solutions to the delay Lyapunov equations (6) and (4). The energies defined by (19) and (18) are for $T \rightarrow \infty$ given in terms of $U_{o}$ and $U_{c}$ by,

$$
E_{o}\left(x_{0}\right):=\lim _{T \rightarrow \infty} E_{o}\left(x_{0}, T\right)=x_{0}^{\mathrm{T}} U_{o}(0) x_{0}
$$

and

$$
E_{c}\left(x_{1}\right):=\lim _{T \rightarrow \infty} E_{c}\left(x_{1}, T\right)=\left\{\begin{array}{cl}
x_{1}^{\mathrm{T}} U_{c}(0)^{\dagger} x_{1} & \text { if } x_{1} \in \operatorname{Im} U_{c}(0) \\
\infty & \text { if } x_{1} \notin \operatorname{Im} U_{c}(0) .
\end{array}\right.
$$

Remark 2 (Notion of state of a time-delay system) Similar to second order systems, the real vector $x(t) \in \mathbb{R}^{n}$ (which we call the current position) does not define the state of a time-delay system since the derivative (1a) depends on a previous position ( $\tau$ time-units ago). The state of a time-delay system must also involve the position at the past $\tau$ time-units.

The energy concepts are changed in an analogous way. In the definition of $E_{o}$, we start with the function, $x(t)=0$ for $t<0$ and $x(0)=x_{0}$, similar to the second order case where (14) was the output corresponding to $x(0)=x_{0}$ and $v(0)=0$. For $E_{c}$, we had for the second order case in (16) that the terminal velocity was free. In analogy, the optimization problem (19) can also be seen as the optimization where the terminal history is a free variable since

$$
E_{c}\left(x_{1}, T\right)=\inf _{x(T+\theta), \theta \in[-\tau, 0)} \inf _{\substack{u \in L_{2}([0, T]), x(T, 0,0, u)=x_{1}}} \int_{0}^{T}\|u(t)\|^{2} d t .
$$

Analogous to the second order case, the optimization problem is a free history optimization problem. 
3.2 Partitioning of infinite-dimensional Gramians

A common way to analyze time-delay systems is to reformulate the system as an infinite dimensional first-order system, using an operator expressed with the notation known as head-tail formulation which we now briefly summarize, following the standard reference [8].

Consider the Hilbert space $X=\mathbb{R}^{n} \times \mathcal{L}_{2}\left([-\tau, 0), \mathbb{R}^{n}\right)$, with the induced scalar product. We define an operator $A$ on $X$ with domain

$$
\mathcal{D}(A):=\left\{\left(\begin{array}{l}
r \\
\varphi
\end{array}\right) \in X \mid \begin{array}{l}
\varphi \text { absolutely continuous on }[-t, 0), \\
\varphi^{\prime} \in \mathcal{L}_{2}\left([-\tau, 0), \mathbb{R}^{n}\right), r=\varphi(0)
\end{array}\right\},
$$

where $\varphi^{\prime}(\theta)=\frac{d \varphi(\theta)}{d \theta}, \theta \in(-\tau, 0)$ and action

$$
A\left(\begin{array}{l}
r \\
\varphi
\end{array}\right):=\left(\begin{array}{c}
A_{0} r+A_{1} \varphi(-\tau) \\
\varphi^{\prime}
\end{array}\right), \quad\left(\begin{array}{l}
r \\
\varphi
\end{array}\right) \in \mathcal{D}(A) .
$$

The delay equation (1) is exponentially stable, if and only if $A$ is the infinitesimal generator of an exponentially stable strongly continuous semigroup $T$. If we further define $B: \mathbb{R}^{m} \rightarrow X$ and $C: X \rightarrow \mathbb{R}^{p}$ by

$$
B u=\left(\begin{array}{c}
B_{0} u \\
0
\end{array}\right), \quad C\left(\begin{array}{l}
x \\
\varphi
\end{array}\right)=C_{0} x,
$$

we can rewrite (1) in first order form as

$$
\left\{\begin{array}{l}
\frac{d}{d t} z_{t}=A z_{t}+B u(t) \\
y(t)=C z_{t}
\end{array}\right.
$$

The connection between a solution of (1) and a corresponding solution of (24) is the following. The state of (24), i.e., $z_{t}$, consists of the vector $x(t)$ and the function segment $x(t+\theta), \theta \in[-\tau, 0)$, corresponding to the trajectory of $x$ at $t$ and the past $\tau$ time units, i.e.,

$$
z_{t}=\left(\begin{array}{c}
x(t) \\
x(t+\cdot)
\end{array}\right)
$$

In words, the state of (24) consists of a "head", which contains the current value of $x$, i.e., the position, and a "tail", which contains the past trajectory of the system over an interval of length $\tau$.

For any $t \geq 0$ the state and the output are given by

$$
\begin{aligned}
z_{t} & =T(t) z_{0}+\int_{0}^{t} T(t-s) B u(s) d s \\
y(t) & =C z_{t} .
\end{aligned}
$$

Following [8, Def. 4.1.20] we define the controllability map $\mathcal{B}^{\infty}$ and the observability $\mathcal{C}^{\infty}$ map by

$$
\begin{aligned}
\mathcal{B}^{\infty} u & =\int_{0}^{\infty} T(t) B u(t) d t, & & \mathcal{B}^{\infty}: L^{2}\left([0, \infty), \mathbb{R}^{m}\right) \rightarrow X \\
\mathcal{C}^{\infty} z & =C T(\cdot) z, & & \mathcal{C}^{\infty}: X \rightarrow L^{2}\left([0, \infty), \mathbb{R}^{p}\right),
\end{aligned}
$$


with the dual operators

$$
\begin{aligned}
& \left(\mathcal{B}^{\infty}\right)^{*} z=B^{*} T(\cdot)^{*} z, \quad\left(\mathcal{B}^{\infty}\right)^{*}: X \rightarrow L^{2}\left([0, \infty), \mathbb{R}^{m}\right) \\
& \left(\mathcal{C}^{\infty}\right)^{*} u=\int_{0}^{\infty} T(t)^{*} C^{*} u(t) d t, \quad\left(\mathcal{C}^{\infty}\right)^{*}: L^{2}\left([0, \infty), \mathbb{R}^{p}\right) \rightarrow X
\end{aligned}
$$

The corresponding Gramians are then $\mathcal{P}^{\infty}=\mathcal{B}^{\infty}\left(\mathcal{B}^{\infty}\right)^{*}$ and $\mathcal{Q}^{\infty}=\left(\mathcal{C}^{\infty}\right)^{*} \mathcal{C}^{\infty}$. In the following reasoning we will also consider the finite-horizon reachability Gramian. For $\theta>0$ let $\mathcal{B}^{\theta}: \mathcal{L}_{2}\left([0, \theta], \mathbb{R}^{m}\right) \rightarrow X$ and its dual be defined by

$$
\mathcal{B}^{\theta} u=\int_{0}^{\theta} T(\theta-t) B u(t) d t \quad \text { and } \quad\left(\mathcal{B}^{\theta}\right)^{*} z=B^{*} T(\theta-\cdot)^{*} z .
$$

Then for the Gramian $\mathcal{P}^{\theta}:=\mathcal{B}^{\theta}\left(\mathcal{B}^{\theta}\right)^{*}$ we have

$$
\mathcal{P}^{\theta}=\int_{0}^{\theta} T(\theta-t) B B^{*} T(\theta-t)^{*} d t=\int_{0}^{\theta} T(t) B B^{*} T(t)^{*} d t \stackrel{\theta \rightarrow \infty}{\longrightarrow} \mathcal{P}^{\infty} .
$$

We partition

$$
\mathcal{P}^{\infty}=\left[\begin{array}{ll}
\mathcal{P}_{11}^{\infty} & \mathcal{P}_{12}^{\infty} \\
\mathcal{P}_{21}^{\infty} & \mathcal{P}_{22}^{\infty}
\end{array}\right] \quad \text { and } \quad \mathcal{Q}^{\infty}=\left[\begin{array}{ll}
\mathcal{Q}_{11}^{\infty} & \mathcal{Q}_{12}^{\infty} \\
\mathcal{Q}_{21}^{\infty} & \mathcal{Q}_{22}^{\infty}
\end{array}\right]
$$

according to $z_{t}$. We will now show that the leading blocks of the partitioned Gramians are equal to the solutions of the corresponding delay Lyapunov equations at $t=0$.

Theorem 2 Consider an exponentially stable time-delay system (1) and let $U_{c}$ and $U_{o}$ be the solutions to the delay Lyapunov equations (6) and (4). Then

$$
\mathcal{Q}_{11}^{\infty}=U_{o}(0)
$$

and

$$
\mathcal{P}_{11}^{\infty}=U_{c}(0) .
$$

Proof In order to show (27) we first express the energy $E_{o}$ (defined in (18)) with the (operator) Gramian $\mathcal{Q}^{\infty}$. From the equivalence of the time-delay system (1) and the infinite-dimensional system (24), it follows from the definition (18) and (26), that for an arbitrary $x_{0} \in \mathbb{R}^{n}$,

$$
E_{o}\left(x_{0}\right)=\int_{0}^{\infty} y(t)^{\mathrm{T}} y(t) d t=\|y\|_{L^{2}}^{2}=\int_{0}^{\infty}\left\langle C T(t) z_{0}, C T(t) z_{0}\right\rangle d t=\left\langle z_{0}, \mathcal{Q}^{\infty} z_{0}\right\rangle,
$$

where

$$
z_{0}=\left[\begin{array}{c}
x_{0} \\
0
\end{array}\right]
$$

Hence, from the partitioning of $\mathcal{Q}^{\infty}$, we have $E_{o}\left(x_{0}\right)=x_{0}^{\mathrm{T}} \mathcal{Q}_{11}^{\infty} x_{0}$. Since, $x_{0}$ was chosen arbitrarily and $\mathcal{Q}_{11}^{\infty}$ is symmetric, the conclusion (27) follows from the characterization of $E_{o}\left(x_{0}\right)$ in $(21)$.

To show (28) and to characterize $E_{c}$, we consider a finite time $\theta>0$ first. Note that $\operatorname{Im} \mathcal{P}^{\theta}$ is the set of all states $z_{1}$ reachable from $z_{0}=0$ in time $\theta$. Let $\mathcal{E}_{c}\left(z_{1}, \theta\right)$ denote the minimal energy needed to reach $z_{1} \in \operatorname{Im} \mathcal{P}^{\theta}$ in time $\theta$. If

$$
z_{1}=\left[\begin{array}{l}
x_{1} \\
\phi_{1}
\end{array}\right]=\mathcal{P}^{\theta}\left[\begin{array}{c}
\xi \\
\psi
\end{array}\right] \quad \text { for some } \quad \xi \in \mathbb{R}^{n}, \psi \in \mathcal{L}_{2}\left([-\tau, 0), \mathbb{R}^{n}\right)
$$


then it follows from an argument analogous to the proof of Lemma 2 that with $z_{1}$, $\xi$ and $\psi$ as above we have

$$
\mathcal{E}_{c}\left(z_{1}, \theta\right)=\left\langle z_{1},\left[\begin{array}{l}
\xi \\
\psi
\end{array}\right]\right\rangle=\left\langle\left[\begin{array}{c}
\xi \\
\psi
\end{array}\right], \mathcal{P}^{\theta}\left[\begin{array}{l}
\xi \\
\psi
\end{array}\right]\right\rangle .
$$

Now take an arbitrary $x_{1} \in \operatorname{Im} P(\theta)$, and recall that $E_{c}\left(x_{1}, \theta\right)$ can be intepreted as the solution of the free-tail optimization problem (in the sense of (23)) and we have

$$
E_{c}\left(x_{1}, \theta\right)=\min _{\phi} \mathcal{E}_{c}\left(\left[\begin{array}{c}
x_{1} \\
\phi
\end{array}\right], \theta\right) \text {. }
$$

Let $\phi_{1}$ be a minimizer.

Now consider a small variation $(\xi+\delta \xi, \psi+\delta \psi)$ of the preimage $(\xi, \psi)$ with the property

$$
\mathcal{P}_{11}^{\theta} \delta \xi+\mathcal{P}_{12}^{\theta} \delta \psi=0
$$

This property implies that the variation does not change $x_{1}$ (in (29)) and we can define $\delta \phi$ by

$$
\left[\begin{array}{c}
x_{1} \\
\phi_{1}+\delta \phi
\end{array}\right]=\mathcal{P}^{\theta}\left[\begin{array}{c}
\xi+\delta \xi \\
\psi+\delta \psi
\end{array}\right]
$$

We will now characterize the minimum (31), by noting that for any sufficiently small $\delta \xi$ and $\delta \psi$, the energy change under the considered variation must vanish to first order, i.e.,

$$
\begin{aligned}
\mathcal{E}_{c}\left(\left[\begin{array}{c}
x_{1} \\
\phi_{1}+\delta \phi
\end{array}\right], \theta\right)-\mathcal{E}_{c}\left(\left[\begin{array}{c}
x_{1} \\
\phi_{1}
\end{array}\right], \theta\right) & \approx 2\left\langle\xi, \mathcal{P}_{11}^{\theta} \delta \xi+\mathcal{P}_{12}^{\theta} \delta \psi\right\rangle+2\left\langle\psi, \mathcal{P}_{21}^{\theta} \delta \xi+\mathcal{P}_{22}^{\theta} \delta \psi\right\rangle \\
& =2\left\langle\left[\begin{array}{c}
\mathcal{P}_{12}^{\theta} \\
\mathcal{P}_{22}^{\theta}
\end{array}\right] \psi,\left[\begin{array}{c}
\delta \xi \\
\delta \psi
\end{array}\right]\right\rangle \stackrel{!}{=} 0
\end{aligned}
$$

Hence $\left[\begin{array}{l}\mathcal{P}_{12}^{\theta} \\ \mathcal{P}_{22}^{\theta}\end{array}\right] \psi \in\left(\operatorname{ker}\left[\mathcal{P}_{11}^{\theta} \mathcal{P}_{12}^{\theta}\right]\right)^{\perp}=\operatorname{Im}\left[\begin{array}{l}\mathcal{P}_{11}^{\theta} \\ \mathcal{P}_{21}^{\theta}\end{array}\right]$, where we exploit that this is a finite-dimensional subspace of $X$ and therefore closed. Thus there exists an $\alpha \in \mathbb{R}^{m}$ with $\left[\begin{array}{c}\mathcal{P}_{11}^{\theta} \\ \mathcal{P}_{21}^{\theta}\end{array}\right] \alpha=\left[\begin{array}{c}\mathcal{P}_{12}^{\theta} \\ \mathcal{P}_{22}^{\theta}\end{array}\right] \psi$, i.e., $\left[\begin{array}{c}\alpha \\ -\psi\end{array}\right] \in \operatorname{ker} \mathcal{P}^{\theta}$.

Therefore we have

$$
\left[\begin{array}{l}
x_{1} \\
\phi_{1}
\end{array}\right]=\mathcal{P}^{\theta}\left[\begin{array}{c}
\xi+\alpha \\
\psi-\psi
\end{array}\right]=\mathcal{P}^{\theta}\left[\begin{array}{l}
\tilde{\xi} \\
0
\end{array}\right],
$$

which implies that $x_{1}=\mathcal{P}_{11}^{\theta} \tilde{\xi}$. We conclude that a position $x_{1}$ is reachable in time $\theta$ if and only if $x_{1} \in \mathcal{P}_{11}^{\theta} \tilde{\xi}$. Hence $\operatorname{Im} P(\theta)=\operatorname{Im} \mathcal{P}_{11}^{\theta}$.

By combining Lemma 2 with (31) and (30) we have

$$
x_{1}^{\mathrm{T}} P(\theta)^{\dagger} x_{1}=\min _{\phi} \mathcal{E}_{c}\left(\left[\begin{array}{c}
x_{1} \\
\phi
\end{array}\right], \theta\right)=\tilde{\xi}^{\mathrm{T}} \mathcal{P}_{11}^{\theta} \tilde{\xi}=x_{1}^{\mathrm{T}}\left(\mathcal{P}_{11}^{\theta}\right)^{\dagger} x_{1}
$$

We conclude that $P(\theta)^{\dagger}=\left(\mathcal{P}^{\theta}\right)^{\dagger}$ since they share the same image and the same kernel, and the quadratic forms coincide on the image. From the uniqueness of the Moore-Penrose-inverse we have $P(\theta)=\mathcal{P}_{11}^{\theta}$ and in the limit we get $U_{c}(0)=\mathcal{P}_{11}^{\infty}$. 
Remark 3 Note that $\mathcal{P}^{\infty}$ and $\mathcal{Q}^{\infty}$ are the unique self-adjoint solutions to the Lyapunov equations

$$
\begin{aligned}
\forall z_{1}, z_{2} \in \mathcal{D}\left(A^{*}\right): & \left\langle\mathcal{P}^{\infty} z_{1}, A^{*} z_{2}\right\rangle+\left\langle A^{*} z_{1}, \mathcal{P}^{\infty} z_{2}\right\rangle=-\left\langle B^{*} z_{1}, B^{*} z_{2}\right\rangle \\
\forall z_{1}, z_{2} \in \mathcal{D}(A): & \left\langle\mathcal{Q}^{\infty} z_{1}, A z_{2}\right\rangle+\left\langle A z_{1}, \mathcal{Q}^{\infty} z_{2}\right\rangle=-\left\langle C z_{1}, C z_{2}\right\rangle
\end{aligned}
$$

where $A^{*}$ is the Hilbert-space adjoint of $A$ [8, Thm. 4.1.23]. A different proof of (27) is provided in [30, Equation (6.19)], based on the corresponding infinitedimensional Lyapunov equation. The same type of reasoning does not seem to carry over to (28).

\section{Truncation based on position balancing}

We have now characterized the Gramians $U_{c}(0)$ and $U_{o}(0)$ by showing that they can be interpreted as an energy quantity representing observability and controllability of a given position. We have shown that they also coincide with the leading blocks in the partitioning of the infinite-dimensional observability and controllability Gramians. Moreover, note that the coordinate transformation (17) corresponds to a similarity transformation of the product of the Gramians, i.e., $\tilde{U}_{c}(0) \tilde{U}_{o}(0)=T U_{c}(0) U_{o}(0) T^{-1}$.

This leads to the following natural definition of a position balanced system.

Definition 2 The time-delay system (1) is called position balanced if

$$
U_{c}(0)=U_{o}(0)=\Sigma,
$$

where $\Sigma$ is diagonal.

Note again that $U_{c}(0)$ and $U_{o}(0)$ undergo a contragredient transformation with the coordinate transformation (17). We can hence follow the standard procedure to balance and truncate a system using a Cholesky decomposition (see e.g. [1]).

Theorem 3 Consider the Cholesky decomposition of the Gramians $U_{c}(0)=S^{\mathrm{T}} S$ and $U_{o}(0)=R^{\mathrm{T}} R$, such that $S$ and $R$ are upper triangular. Let $U, \Sigma, V$ be the singular value decomposition of $S R^{\mathrm{T}}=U \Sigma V^{\mathrm{T}}$ and define the coordinate transformation as $T=\Sigma^{-1 / 2} V^{\mathrm{T}} R$ and $T^{-1}=S^{\mathrm{T}} U \Sigma^{-1 / 2}$. Let $\tilde{U}_{c}$ and $\tilde{U}_{o}$ be the solution to the delay Lyapunov equations for the transformed time-delay system, $\tilde{A}_{0}=T A_{0} T^{-1}, \tilde{A}_{1}=$ $T A_{1} T^{-1}, \tilde{B}_{0}=T B_{0}$ and $\tilde{C}_{0}=C_{0} T^{-1}$. Then,

$$
\tilde{U}_{c}(0)=\tilde{U}_{o}(0)=\left(\begin{array}{ccc}
\sigma_{1} & & \\
& \ddots & \\
& & \sigma_{n}
\end{array}\right)=\Sigma \text {. }
$$

That is, the transformed system is position balanced. 
Algorithm 1 Model reduction based on position balancing

1: Compute $U_{c}(0)$ and $U_{o}(0)$, e.g., using a numerical scheme [11],[33] or explicit formula $[30],[12]$.

2: Compute the Cholesky decomposition of $U_{c}(0)$ and $U_{o}(0)$, i.e., upper triangular $R$ and $S$ such that

$$
U_{c}(0)=S^{\mathrm{T}} S \text { and } U_{o}(0)=R^{\mathrm{T}} R \text {. }
$$

3: Compute the truncated singular value decomposition of $S R^{\mathrm{T}}$, i.e., if $S R^{\mathrm{T}}=U \Sigma V^{\mathrm{T}}$ with diagonal $\Sigma$ ordered by decreasing magnitude, we compute $U, \hat{V} \in \mathbb{R}^{n \times r}$ as the $r$ first columns of $U$ and $V$ and $\hat{\Sigma} \in \mathbb{R}^{r \times r}$ as the leading block of $\Sigma$.

4: Compute $T_{1}^{\mathrm{T}}=\hat{\Sigma}^{-1 / 2} \hat{V}^{\mathrm{T}} R \in \mathbb{R}^{r \times n}$ and $T_{2}=S^{\mathrm{T}} \hat{U} \hat{\Sigma}^{-1 / 2} \in \mathbb{R}^{n \times r}$

5: Compute reduced model $\hat{A}_{0}=T_{1}^{\mathrm{T}} A_{0} T_{2}, \hat{A}_{1}=T_{1}^{\mathrm{T}} A_{1} T_{2}, \hat{B}_{0}=T_{1}^{\mathrm{T}} B_{0}$ and $\hat{C}_{0}=C_{0} T_{2}$.

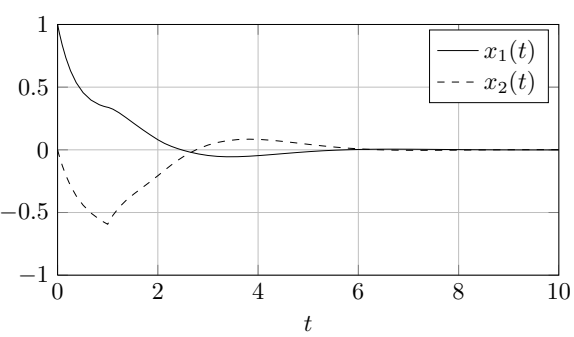

(a) $x$ with $u(t)=0$

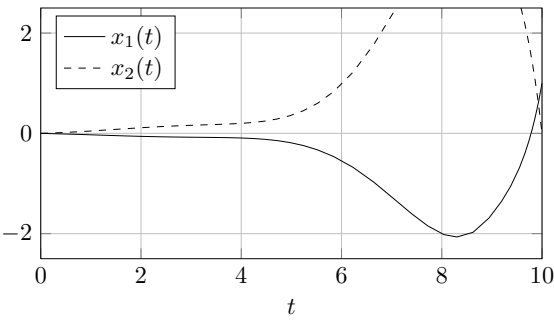

(c) $x$ with (32) and $x(T)=e_{1}$

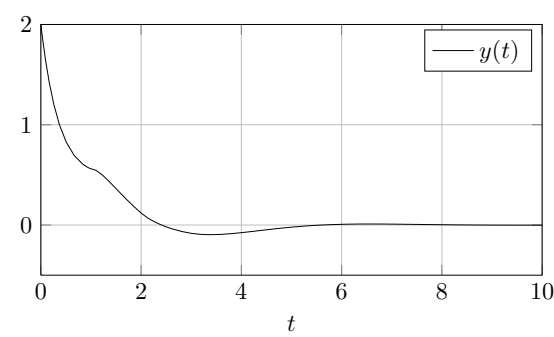

(b) Output with $u(t)=0$

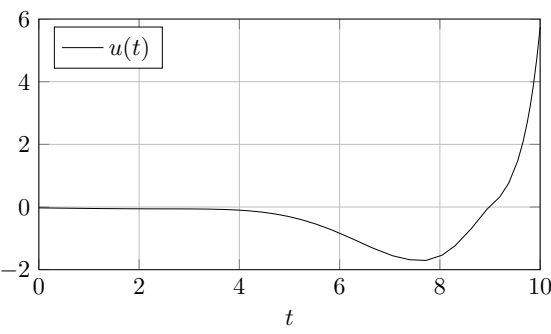

(d) Input $u(t)$ with (32)

Fig. 1 Simulations with the unbalanced system for the example in Section 5.1.

\section{Examples}

\subsection{Illustration of balancing}

Consider the time-delay system given by

$$
A_{0}=\left(\begin{array}{cc}
-2 & -1 \\
-3 / 2 & -1 / 2
\end{array}\right), \quad A_{1}=\left(\begin{array}{cc}
0 & 1 / 2 \\
1 & 0
\end{array}\right), \quad B_{0}=\left(\begin{array}{c}
1 \\
-1
\end{array}\right), \quad C_{0}^{\mathrm{T}}=\left(\begin{array}{c}
2 \\
0.2
\end{array}\right)
$$




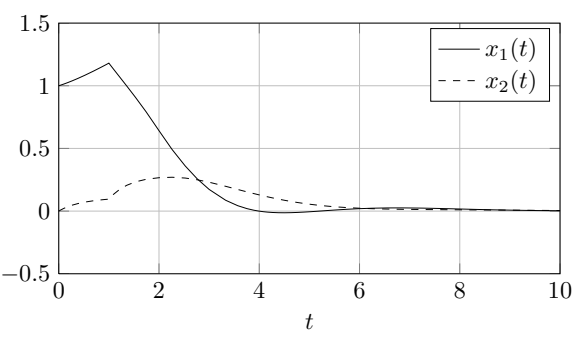

(a) $x$ with $u(t)=0$

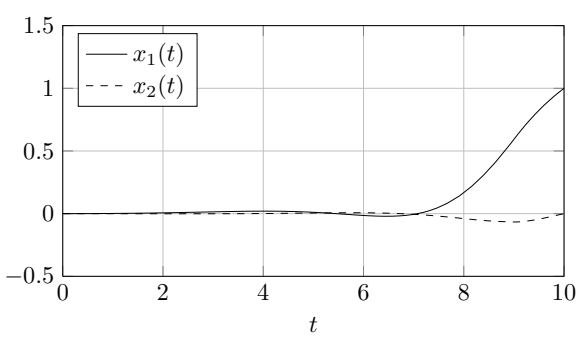

(c) $x$ with $(32)$ and $x(T)=e_{1}$

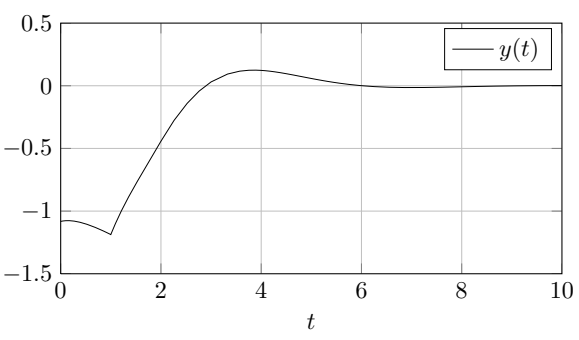

(b) Output with $u(t)=0$

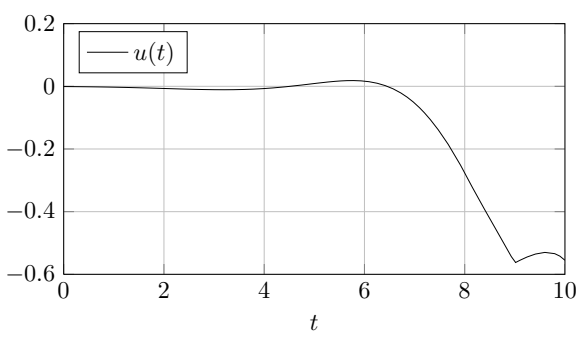

(d) Input $u(t)$ with $(32)$

Fig. 2 Simulations with the balanced system for the example in Section 5.1

The system is stable and the spectral abscissa is $\alpha \approx-0.52$. We will need $U_{c}(0)$ and $U_{o}(0)$ for this system which were computed to,

$$
U_{c}(0) \approx\left(\begin{array}{cc}
0.93 & -1.74 \\
-1.74 & 3.63
\end{array}\right), U_{o}(0) \approx\left(\begin{array}{cc}
1.27 & -0.41 \\
-0.41 & 0.37
\end{array}\right) .
$$

In a first simulation, we start the time-delay system with $x(0)=e_{1}=(1,0)^{\mathrm{T}}$ and $x(t)=0$ for $t<0$ and let $u(t)=0$. This is a standard delay-differential equation and we can integrate it with standard software for integration of delaydifferential equations. The evolution of $x(t)$ until $t=T=10$ is given in Fig. 1a. The numerical integral of $|y(t)|^{2}$ is given in Table 1 . Note that $e_{1}^{\mathrm{T}} U_{o}(0) e_{1} \approx \int_{0}^{\mathrm{T}}|y(t)|^{2} d t$ as predicted by Theorem 1 .

Lemma 2 gives a formula for the input which steers the system optimally to a given position in time $t=T$. We hence expect that the input

$$
u(t)=B_{0}^{\mathrm{T}} K(T-t)^{\mathrm{T}} U_{c}(0)^{-1} e_{1}
$$

will steer the system from $x(0)=0$ to $x(T)=e_{1}$ in a close to optimal manner for sufficiently large $T$. Note that $P(T) \approx U_{c}(0)$ for sufficiently large $T$ since $K(t)$ decays asymptotically exponentially with rate $e^{\alpha t}$.

In order to evaluate the input function $u(t)$ we need to evaluate the fundamental solution $K(t)$. This can be done numerically, e.g., by noting that the vectorized 


\begin{tabular}{|c|c|c|c|}
\hline \multicolumn{2}{|c|}{ Unbalanced } & \multicolumn{2}{c|}{ Balanced } \\
\hline \hline$E_{c}\left(e_{1}, T\right)$ & $E_{o}\left(e_{1}, T\right)$ & $E_{c}\left(e_{1}, T\right)$ & $E_{o}\left(e_{1}, T\right)$ \\
\hline \hline 11.03 & 1.27 & 0.50 & 1.98 \\
\hline
\end{tabular}

Table 1 The energies before and after the balancing process for the example in Section 5.1. Note that for the balanced system $E_{c}\left(e_{1}, T\right) \approx 1 / E_{o}\left(e_{1}, T\right)$.

equation $\operatorname{vec}(K(t))$ started with $\operatorname{vec}(K(0))=\operatorname{vec}(I)$ is a standard delay-differential equation and we can again use software for solving delay-differential equation. We now start the algorithm with $x(t)=0$ for $t \leq 0$ and use the control (32) and again integrate until $T=10$. We clearly see in Fig. 1c-d that the control steers the system to $x(t)=e_{1}$. The integral of the square of the input is given in Table 1 , i.e., consistent with Theorem 1 in the sense that $e_{1}^{\mathrm{T}} U_{c}(0)^{-1} e_{1} \approx \int_{0}^{T}\|u(t)\|^{2} d t$.

Note that the system is not position balanced in the energy sense since $E_{c}\left(e_{1}, T\right) \neq$ $1 / E_{o}\left(e_{1}, T\right)$. By carrying out the balancing process in Theorem 2 we get a new system

$$
\tilde{A}_{0} \approx\left(\begin{array}{cc}
0.13 & 0.71 \\
0.24 & -2.63
\end{array}\right), \quad \tilde{A}_{1} \approx\left(\begin{array}{cc}
-0.74 & -0.10 \\
0.43 & 0.74
\end{array}\right), \quad \tilde{B}_{0} \approx\left(\begin{array}{c}
-1.1 \\
0.65
\end{array}\right), \quad \tilde{C}_{0}^{\mathrm{T}} \approx\left(\begin{array}{c}
-1.08 \\
0.95
\end{array}\right) .
$$

After balancing both delay Lyapunov are equal and diagonal at $\theta=0$,

$$
\tilde{U}_{c}(0)=\tilde{U}_{o}(0)=\Sigma \approx\left(\begin{array}{cc}
1.98 & 0 \\
0 & 0.16
\end{array}\right) .
$$

The same simulations described for the unbalanced system are now carried out for this balanced system and the results are presented in Fig. 2. In the balanced system, $E_{c}\left(e_{1}, T\right) \approx 1 / E_{o}\left(e_{1}, T\right)$ consistent with the interpretation that a position should be equally difficult to observe as it is to reach. This is observed in Table 1.

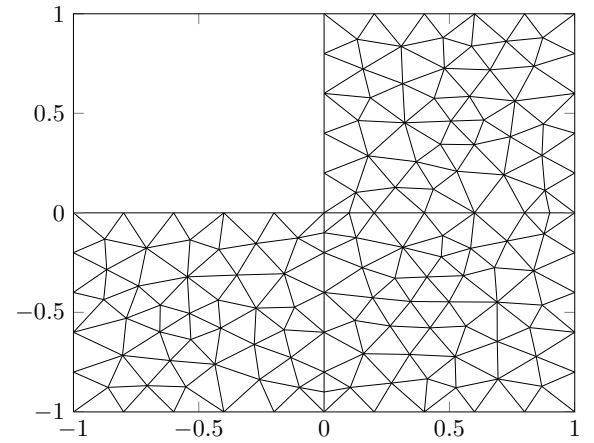

(a)

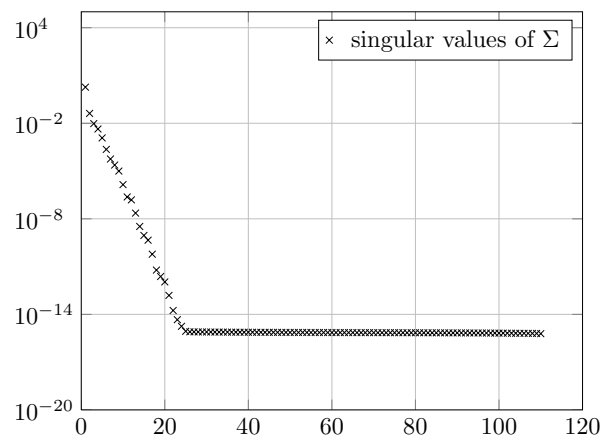

(b)

Fig. 3 The triangularized L-shaped domain and singular values of $\Sigma$ for balanced system for the example in Section 5.2 . 


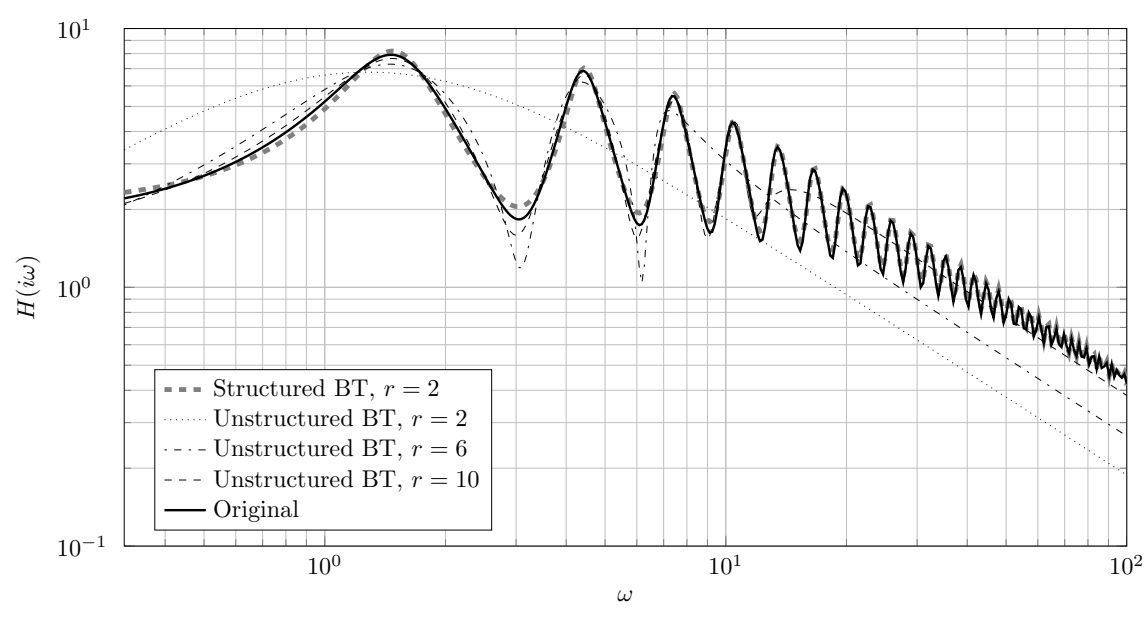

Fig. 4 Transfer function and error for the proposed method and an unstructured approach for the example in Section 5.2

\subsection{Illustration of truncation}

In order to illustrate the truncation based on position balancing, we will now consider a time-delay system stemming from the discretization of a partial differential equation. We let $A_{0}$ be the matrix corresponding to the discretization of the PDE eigenvalue problem

$$
-\Delta u=\lambda u
$$

on the L-shaped domain Figure 3a with Dirichlet boundary conditions and the grid also given in Figure 3a. The discretization is such that $n=110$. We let $A_{1}=$ $\beta e_{j} e_{k}^{\mathrm{T}}$ where $j$ and $k$ corresponding to the grid points closest to $(-0.4,-0.6)$ and $(0.2,-0.6)$, respectively, localizing the delay-term in space. With this construction $\left\|A_{0}\right\|_{2}=6.0$ and $\left\|A_{1}\right\|_{2}=\beta=5$. We fixed the delay to $\tau=2$, and $B_{0}^{\mathrm{T}}=C_{0}=$ $(1, \ldots, 1)$, corresponding to an average of $u$ in the whole domain. The system is stable with a spectral abscissa $\alpha \approx-0.4$.

In Algorithm 1 we need to compute $U_{c}(0)$ and $U_{o}(0)$ for a system with $n=110$. For this we use a discretization approach which has been used in another setting [33]. We discretize the infinitesimal generator with a spectral method, which results in approximating (1) with a linear system without delay but with larger order. The discretization approach involves a choice of the number of discretization points. We chose this number by increasing it until no substantial change in the reduction was observed. In this example, $N=20$ was sufficient.

The singular values are shown in Fig. 3. Note that the boundary condition in the delay Lyapunov equation, i.e., (4c) and (6c), is a (standard) Lyapunov equation in $U_{c}(0)$ and $U_{o}(0)$ with low rank right-hand side. This explains the fast decay of the singular values, compare e.g. [1].

The result of Algorithm 1 is visualized in Figure 4 and Figure 5.

In Figure 4 we observe that the oscillations of the transfer function are well matched in the structured reduced model. In order to show the advantage of a structured approach, we also carried out simulations which were not preserving 
the structure. Similar to the procedure that we used to compute $U_{c}(0)$ and $U_{o}(0)$ we can discretize the system, and get a large linear system. This can subsequently be reduced with the standard version of balanced truncation for linear dynamical systems without delay. This is also shown in Figure 4. Note that only a finite number of oscillations is matched in the unstructured approach.

In Figure 5 we observe that the error decreases with the size of the reduced model. This is consistent with the decay of the singular values in Fig. 3.

However, different from the case of full-state balanced truncation of systems without delay, the error is not of the same magnitude as the largest neglected singular value. In particular, for $r=25$, the neglected singular values are numerically equal to zero. But the error is still significantly larger than the unit roundoff error.

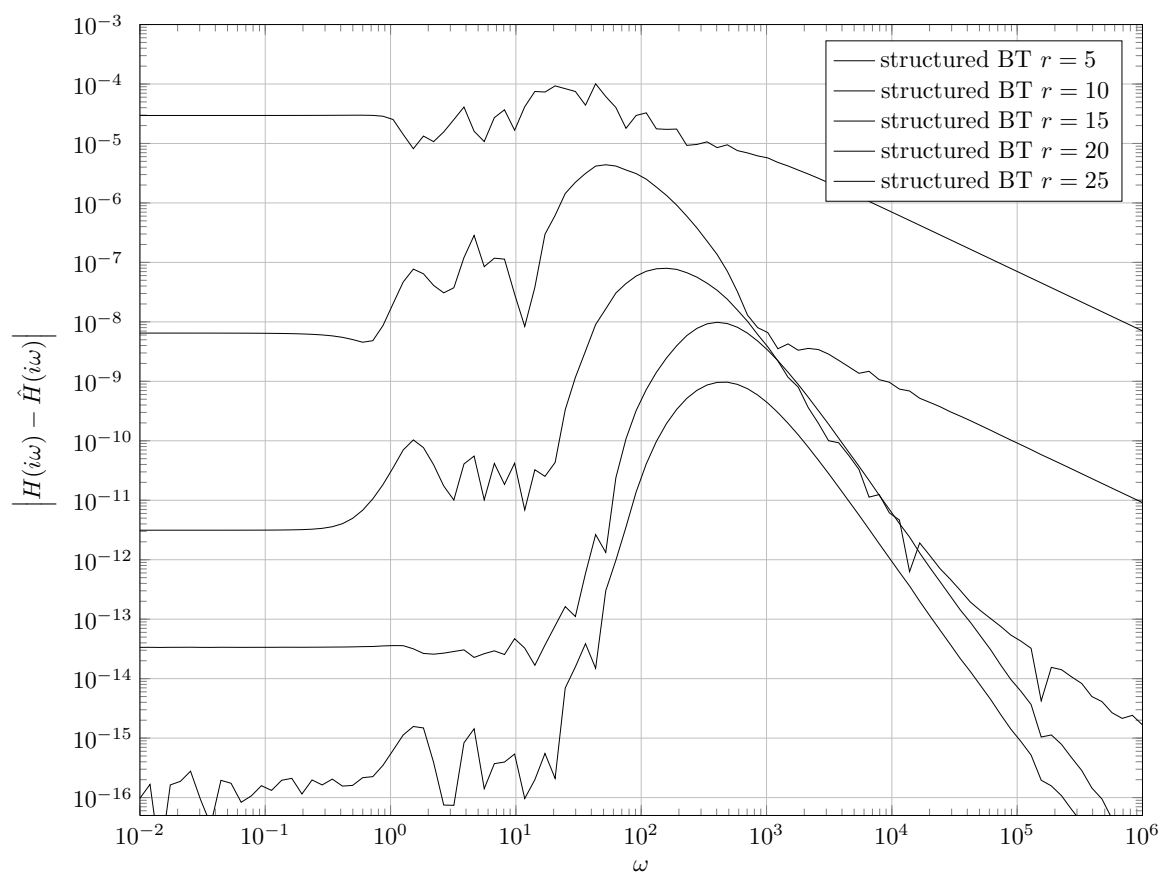

Fig. 5 Error of the truncation based on position balancing for the example in Section 5.2.

\section{Concluding remarks}

We have here suggested a variant of balanced truncation for delay systems. The computationally dominating part of the algorithm is the computation of $U_{c}(0)$ and $U_{o}(0)$, which stem from the generalization of the Lyapunov equation. Although computational aspects of the delay Lyapunov equation have received some attention (e.g. [11],[12]), there is, for instance, no method similar or as efficient as the Bartels-Stewart algorithm [3] available for the delay Lyapunov equation. 
Also note that some generalizations of the results in this paper are straightforward, because delay Lyapunov equations have already been studied for many variant of time-delay systems. An important component in the derivation of our result is the connection between the definition of $U_{c}$ (equation (5)) and the delay Lyapunov equations (6). This connection has, for instance, been worked out for multiple delays [17], neutral systems [30],[14], and time-delay systems with distributed delays [15].

We finally wish to point out that, although our approach is natural in terms of the energy concepts and Gramians, it does share some of the disadvantages observed for a similar approach for second order systems in [7],[22],[31]. In particular, stability is not always preserved and error bounds are not available. On the other hand, it yields a computable method (at least for moderate dimensions), which preserves the structure of the system and performs quite convincing in numerical examples. In contrast to this, a full state balancing approach would lead to infinite-dimensional operator equations, which are difficult and expensive to solve, and typically do not lead to a reduced system of the form (2).

\section{Acknowledgements}

This work has been supported by the Programme of Interuniversity Attraction Poles of the Belgian Federal Science Policy Office (IAP P6- DYSCO), by OPTEC, the Optimization in Engineering Center of the K.U. Leuven, and by the project STRT1-09/33 of the K.U. Leuven Research Council.

\section{References}

1. Antoulas, A.: Approximation of large-scale dynamical systems. SIAM (2005)

2. Antoulas, A., Sorensen, D., Gugercin, S.: A survey of model reduction methods for largescale systems. Contemp. Math. 280, 193-219 (2001)

3. Bartels, R., Stewart, G.W.: Solution of the matrix equation $A X+X B=C$. Comm A.C.M. 15(9), 820-826 (1972)

4. Beattie, C., Gugercin, S.: Interpolatory projection methods for structure-preserving model reduction. Syst. Control Lett. 58(3), 225-232 (2009)

5. Benner, P., Mehrmann, V., Sorensen, D. (eds.): Dimension Reduction of Large-Scale Systems. Springer-Verlag (2005)

6. Benner, P., Saak, J.: Efficient balancing based MOR for large scale second order systems. Math. Comput. Model. Dyn. Syst. 17(2), 123-143 (2011)

7. Chahlaoui, Y., Lemonnier, D., Vandendorpe, A., Dooren, P.V.: Second-order balanced truncation. Linear Algebra Appl. 415, 373-384 (2006)

8. Curtain, R.F., Zwart, H.: An introduction to infinite-dimensional linear systems theory. Springer-Verlag, NY (1995)

9. Hale, J., Verduyn Lunel, S.M.: Introduction to functional differential equations. SpringerVerlag (1993)

10. Harkort, C., Deutscher, J.: Krylov subspace methods for linear infinite-dimensional systems. IEEE Trans. Autom. Control 56(2), 441-447 (2011)

11. Huesca, E., Mondié, S., Santos, J.: Polynomial approximations of the Lyapunov matrix of a class of time delay systems. In: Proceedings of the 8th IFAC workshop on time-delay systems, Sinaia, Romania (2009)

12. Jarlebring, E., Vanbiervliet, J., Michiels, W.: Characterizing and computing the $\mathcal{H}_{2}$ norm of time-delay systems by solving the delay Lyapunov equation. IEEE Trans. Autom. Control 56(4), 814-825 (2011)

13. Kharitonov, V.: Lyapunov-Krasovskii functionals for scalar time delay equations. Syst. Control Lett. 51(2), 133-149 (2004) 
14. Kharitonov, V.: Lyapunov functionals and Lyapunov matrices for neutral type time delay systems: a single delay case. Int. J. Control 78(11), 783-800 (2005)

15. Kharitonov, V.: Lyapunov matrices for a class of time delay systems. Syst. Control Lett. 55(7), 610-617 (2006)

16. Kharitonov, V., Hinrichsen, D.: Exponential estimates for time delay systems. Syst. Control Lett. 53(5), 395-405 (2004)

17. Kharitonov, V., Plischke, E.: Lyapunov matrices for time-delay systems. Syst. Control Lett. 55(9), 697-706 (2006)

18. Kharitonov, V., Zhabko, A.P.: Lyapunov-Krasovskii approach to the robust stability analysis of time-delay systems. Automatica 39(1), 15-20 (2003)

19. Mäkilä, P., Partington, J.: Laguerre and Kautz shift approximations of delay systems. Int. J. Control 72(10), 932-946 (1999)

20. Mäkilä, P., Partington, J.: Shift operator induced approximations of delay systems. SIAM J. Control Optimization 37(6), 1897-1912 (1999)

21. Mastinšek, M.: Adjoints of solution semigroups and identifiability of delay differential equations in Hilbert spaces. Acta Math. Univ. Comenianae LXIII(2), 193-206 (1994)

22. Meyer, D.G., Srinivasan, S.: Balancing and model reduction for second-order form linear systems. IEEE Trans. Autom. Control 41(11), 1632-1644 (1996)

23. Michiels, W.: Stability and stabilization of time-delay systems. Ph.D. thesis, Katholieke universiteit Leuven (2002)

24. Michiels, W., Jarlebring, E., Meerbergen, K.: Krylov based model order reduction of timedelay systems. Tech. rep., Dept. Comp. Sci., KU Leuven (2010). Accepted for publication in SIAM J. Matrix Anal. Appl.

25. Niculescu, S.I.: Delay effects on stability. A robust control approach. Springer-Verlag London (2001)

26. Ochoa, G., Kharitonov, V.: Lyapunov matrices for neutral type time delay systems. In: Proceedings of the 2nd International Conference on Electrical and Electronics Engineering, Mexico City, Mexico (2005)

27. Ochoa, G., Velázquez-Velázquez, J., Kharitonov, V., Mondié, S.: Lyapunov matrices for neutral type time delay systems. In: Proceedings of the 7th IFAC workshop on time delay systems, Nantes, France (2007)

28. Ouellette, D.V.: Schur complements and statistics. Linear Algebra Appl. 36, 187-295 (1981)

29. Partington, J.: Model reduction of delay systems. In: V. Blondel, A. Megretski (eds.) Unsolved Problems in Mathematical Systems and Control Theory, pp. 29-32. Princeton university press (2004)

30. Plischke, E.: Transient effects of linear dynamical systems. Ph.D. thesis, Universität Bremen (2005)

31. Reis, T., Stykel, T.: Balanced truncation model reduction of second-order systems. Math. Comput. Model. Dyn. Syst. 14(5), 391-406 (2008)

32. Saadvandi, M., Meerbergen, K., Jarlebring, E.: On dominant poles and model reduction of second order time-delay systems. Tech. rep., Dept. Comp. Sci., KU Leuven (2010). Submitted

33. Vanbiervliet, J., Michiels, W., Jarlebring, E.: Using spectral discretisation for the optimal $\mathcal{H}_{2}$ design of time-delay systems. Int. J. Control 84(2), 228-241 (2011)

34. Velázquez-Velázquez, J., Kharitonov, V.: Lyapunov-Krasovskii functionals for scalar neutral type time delay equation. Syst. Control Lett. 58(1), 17-25 (2009)

35. Yan, B., Tan, S., McGaughy, B.: Second-order balanced truncation for passive order reduction of RLCK circuits. IEEE Trans. Circuits Syst., II, Analog Digit. Signal Process. 55, 942-946 (2008)

36. Zigic, D., Watson, L., Beattie, C.: Contragredient transformations applied to optimal projection equations. Linear Algebra Appl. 188-189, 665-676 (1993) 